\title{
Artificial Intelligence as a maturing and growing technology: An urgent need for intelligent systems
}

\author{
X. Yuan ${ }^{\mathrm{a}, *}$ and M. Elhoseny ${ }^{\mathrm{a}, \mathrm{b}}$ \\ ${ }^{a}$ Department of Computers Science and Engineering, Computer Vision and Intelligent Systems Lab, \\ University of North Texas, Denton, TX, USA \\ ${ }^{\mathrm{b}}$ Faculty of Computers and Information, Mansoura University, Egypt
}

\section{Summary}

Artificial intelligence (AI) has come ai long way from the introduction of fuzzy logic by Zadeh, the applications of Norbert Wiener's feedback mechanisms in intelligent systems by Newell and Simon, and John McCarthy's tireless efforts that promoted research on AI. Today, as are emerging to influence many aspects of our lives (from robotics, self-driving cars, and voice recognition systems to medical applications such as healthcare delivery services), businesses and government institutions are deploying and integrating AI into their business process at large scales. So, it's no wonder that the demand for AI professionals in the job market is so high. At the same time, $\mathrm{AI}$ is being integrated into commercial products and services that we use in our daily lives. However, AI is still a maturing and growing technology, and making AI more robust requires extensive research, studies, and innovative ideas.
The accepted papers explore diverse modelling approaches and a number of novel methods and approaches which are proposed and applied to a wide range of AI applications, such as voice recognition, machine learning application, intelligent learning systems, and supported vector machine. Besides, a variety of applications have been considered to show the emerging methodologies of $\mathrm{AI}$ in different fields such as, smart education, business intelligence, image processing, and robotics. Moreover, the special issue focusses on an urgent research topic related to applying $\mathrm{AI}$ techniques on computer communication and network security area.

Finally, we hope that the readers will find all papers in this special issue interesting and useful. The Guest editors wish to thank all the authors and reviewers that contributed to this special issue, and to the Editor-inChief and editorial office of the JIFS for their support.

\footnotetext{
${ }^{*}$ Corresponding author. X. Yuan, Department of Computers Science and Engineering, Computer Vision and Intelligent Systems Lab, University of North Texas, Denton, TX, USA. E-mail: xiaohui.yuan@unt.edu.
} 